\title{
Differential Feedback Modulation of Center and Surround Mechanisms in Parvocellular Cells in the Visual Thalamus
}

\author{
Helen E. Jones, ${ }^{1}$ Ian M. Andolina, ${ }^{1}$ Bashir Ahmed, ${ }^{1}$ Stewart D. Shipp, ${ }^{1}$ Jake T. C. Clements, ${ }^{1}$ Kenneth L. Grieve, ${ }^{2}$ \\ Javier Cudeiro, ${ }^{3}$ Thomas E. Salt, ${ }^{1}$ and Adam M. Sillito ${ }^{1}$ \\ ${ }^{1}$ Department of Visual Neuroscience, UCL Institute of Ophthalmology, University College London, London EC1V 9EL, United Kingdom, ${ }^{2}$ Faculty of Life \\ Sciences, University of Manchester, Manchester M13 9PT, United Kingdom, and ${ }^{3}$ Department of Medicine-Neurocom and INIBIC (Institute of Biomedical \\ Research of A Coruña), University of A Coruña, 15006 A Coruña, Spain
}

\begin{abstract}
Many cells in both the central visual system and other sensory systems exhibit a center surround organization in their receptive field, where the response to a centrally placed stimulus is modified when a surrounding area is also stimulated. This can follow from laterally directed connections in the local circuit at the level of the cell in question but could also involve more complex interactions. In the lateral geniculate nucleus (LGN), the cells relaying the retinal input display a concentric, center surround organization that in part follows from the similar organization characterizing the retinal cells providing their input. However, local thalamic inhibitory interneurons also play a role, and as we examine here, feedback from the visual cortex too. Here, we show in the primate (macaque) that spatially organized cortical feedback provides a clear and differential influence serving to enhance both responses to stimulation within the center of the receptive field and the ability of the nonclassical surround mechanism to attenuate this. In short, both center and surround mechanisms are influenced by the feedback. This dynamically sharpens the spatial focus of the receptive field and introduces nonlinearities from the cortical mechanism into the LGN.
\end{abstract}

\section{Introduction}

In the central visual system the number of "feedback connections" from higher to lower levels often exceed the ascending connections. This is particularly clear in the case of the feedback from layer 6 of the primary visual cortex (V1) to the lateral geniculate nucleus (LGN) (Sillito et al., 2006; Briggs and Usrey, 2011). Despite the anatomical clarity and a range of studies probing the potential role of feedback from V1 to LGN (Tsumoto et al., 1978; McClurkin and Marrocco, 1984; Murphy and Sillito, 1987; Cudeiro and Sillito, 1996; Wörgötter et al., 1998; Przybyszewski et al., 2000; Rivadulla et al., 2002; Webb et al., 2002; Wang et al., 2006; Andolina et al., 2007; Briggs and Usrey, 2009, 2011), we still lack a consensus regarding the primary patterns of effect. There are suggestions of functional selectivity in the role of this feedback in the cat (Tsumoto et al., 1978; Murphy and Sillito, 1987; Gulyás et al., 1990; Cudeiro and Sillito, 1996; Wörgötter et al., 1998; Rivadulla et al., 2002; Wang et al., 2006; Andolina et al., 2007). In the primate there is less direct evidence but compelling grounds for the view that the cortical feedback is subdivided into

\footnotetext{
Received Feb. 20, 2012; revised Sept. 2, 2012; accepted Sept. 6, 2012.

Author contributions: H.E.J., I.M.A., and A.M.S. designed research; H.E.J., I.M.A., B.A., S.D.S., J.T.C.C., K.L.G., J.C., T.E.S., and A.M.S. performed research; H.E.J., I.M.A., B.A.,S.D.S., J.T.C.C., K.L.G., J.C., T.E.S., and A.M.S. analyzed data; H.E.J. and A.M.S. wrote the paper.

This work was supported by Medical Research Council Grant G0701535. We thank Janssen-Cilag Ltd for the kind gift of Sufenta Forte.

Correspondence should be addressed to Helen E. Jones, Department of Visual Neuroscience, UCL Institute of Ophthalmology, 11-43 Bath Street, London EC1V 9EL, UK. E-mail: hjones@ioores.co.uk.

B. Ahmed's present address: Department of Physiology, Anatomy and Genetics, University of Oxford, Oxford 0X1 3QX, UK.

DOI:10.1523/JNEUROSCI.0831-12.2012

Copyright $\odot 2012$ the authors $\quad 0270-6474 / 12 / 3215946-06 \$ 15.00 / 0$
}

three parallel streams subserving the magno-, parvo-, and konicellular channels (Briggs and Usrey, 2009, 2011). Studies cooling or ablating the visual cortex indicated that feedback may increase the gain and magnitude of primate LGN cell responses and enhance the influence of the suppressive surround (Przybyszewski et al., 2000; Webb et al., 2002). On the other hand there is evidence that the extra classical surround suppression in primate LGN cells is actually set up in the retina (Alitto and Usrey, 2008). In general there is a paradox that responses of many LGN cells look remarkably like those of the retinal ganglion cells despite the complex circuitry impinging on them via local synaptic circuits and cortical feedback. The challenge here concerns the class of influence that might be expected from this circuitry and the feedback. The feedback connections are precisely retinotopically organized and this suggests the potential for a focused influence that could follow the resolution of the cortical map.

Rather than attempt to probe the role of feedback by comparing the responses of LGN cells in the presence or absence of a global inactivation of V1 by procedures such as ablation, cooling or TMS (McClurkin and Marrocco, 1984; Murphy and Sillito, 1987; de Labra et al., 2007) we have taken a different route and examined the possibility that the feedback may be focal and spatially organized. This is the first time this approach to dissecting feedback from V1 to the LGN has been attempted in the primate. Our experiments have examined the effect of focal, discrete and reversible pharmacological inactivation of layer 6 cells at retinotopic locations either overlapping or displaced from the receptive fields of simultaneously recorded LGN cells (Fig. 1A). We have restricted our study to parvocellular LGN cells and focused on the spatial properties of their receptive fields. 
A

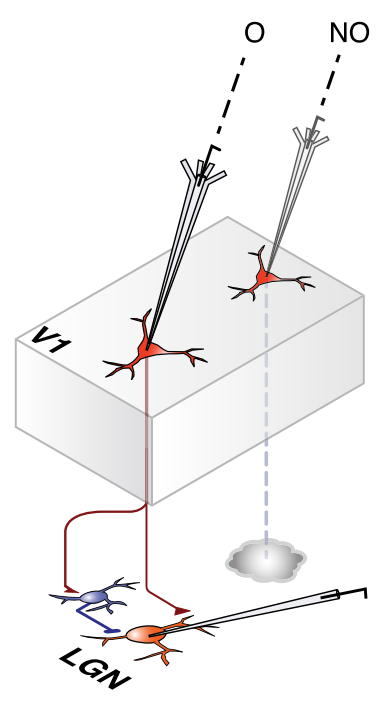

B

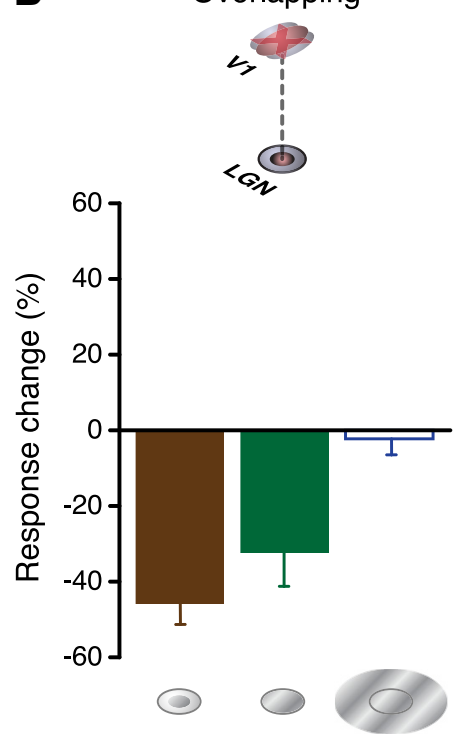

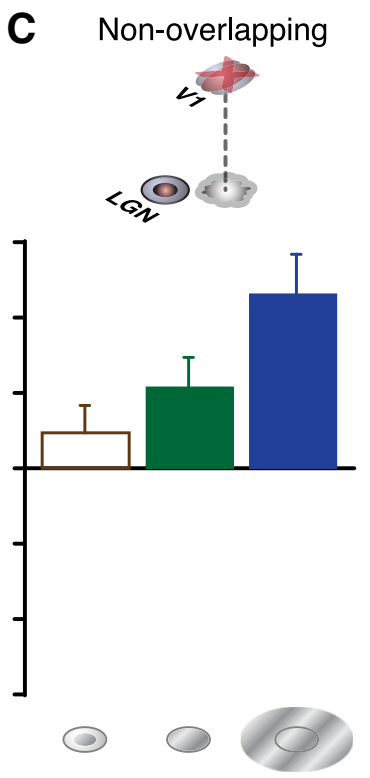

Figure 1. Differential effects of focal inactivation of corticothalamic feedback by iontophoretic application of GABA on overlapping and nonoverlapping LGN cell locations. $A$, Schematic summary of experimental method. Drug inactivation electrode is shown inserted in either an overlapping (0) or nonoverlapping (NO) retinotopic location with respect to that of the LGN. $\boldsymbol{B}, \boldsymbol{C}$, Population summary histograms plot the mean percentage change in $\mathrm{LGN}$ cell responses for overlapping $(B, n=14)$ and nonoverlapping $(C, n=12)$ recording sites for smaller than optimal (left), optimal (middle), and larger than optimal (right) stimuli, across our sample. Error bars indicate SEM. Significant response changes (see Results) denoted by filled bars, nonsignificant changes by open bars.

\section{Materials and Methods}

Experiments were performed on four anesthetized [sufentanil $(4-8$ $\mu \mathrm{g} \cdot \mathrm{kg}^{-1} \cdot \mathrm{h}^{-1}$ in a glucose-enriched Hartmann's solution) and a mixture of $70 \% \mathrm{~N}_{2} \mathrm{O}$ and $30 \% \mathrm{O}_{2}$ supplemented, as required, with halothane $(0.1-0.5 \%)]$ and paralyzed $\left(0.1 \mathrm{mg} \cdot \mathrm{kg}^{-1} \cdot \mathrm{h}^{-1}\right.$ vecuronium bromide) female primates (Macaca mulatta) as previously described (Jones et al., 2001). All procedures were in accordance with British Home Office license requirements and approved by the local ethical review committee at UCL's Institute of Ophthalmology. We recorded single-unit extracellular responses from parvocellular LGN cells using multielectrode arrays of tungsten in glass electrodes and also recorded extracellular responses from single units in V1 using a tungsten-recording electrode glued to a five barreled glass iontophoretic pipette. Our multielectrode arrays were preconfigured to optimally sample from parafoveal parvocellular LGN cells with a predictable retinotopic relationship to the cortical electrode's location. We did not attempt to extend the electrode penetrations down to the ventrally located magnocellular layers given the oblique anatomical arrangement of projection columns through the macaque LGN, but rather focused our efforts to record from a sufficiently sized parvocellular cell population. In preliminary tests, we determined the receptive field properties of all cells using a battery of tests. Cells were identified as parvocellular LGN cells based on physiological response properties, electrode depth, and stereotypical shifts in eye preference and subsequent histological reconstruction of electrode tracks (Wiesel and Hubel, 1966; Jones et al., 2001; McAlonan et al., 2008). Receptive fields were first hand-mapped with bars and spots of light. Then response properties and dimensions were quantitatively mapped using flashing spots (or bars) of light, drifting bars and/or edges and patches of sinusoidal grating. Before running our experimental protocol, we carefully documented the precise location and spatial extent of the receptive field center by exploring the spatial distribution of locations from which a contrast modulated patch or a patch of drifting grating elicited responses. A variety of patch sizes $\left(0.1-0.5^{\circ}\right)$ were used for this test (XY patch). They were presented in a randomized sequence over a set of spatial locations defined in rectangular coordinates. The location giving the largest response was used to define the center location, and the coordinates of our display were adjusted to match this to the display center. This involved several iterations with variations of patch size and display coordinates to optimize both centering and assessment of spatial extent. We then plotted area- summation tuning curves using patches of drifting grating of varying diameters presented in a randomized, interleaved manner.

We then used focal micro-iontophoretic drug application of GABA $(0.5 \mathrm{M}, \mathrm{pH} 3)$ to reversibly inactivate the responses of layer 6 cells in $\mathrm{V} 1$ and explored the effects of this on the responses of the LGN cells to patches of drifting gratings of varying diameter moving in the layer 6 cells preferred orientation and direction. Stimulus diameters were randomly interleaved and we typically presented three to five stimulus cycles of each diameter, repeated over 5-10 trials. The spatial frequency was normally 3 cycles/degree (cpd; range 1-3 cpd), temporal frequency $3 \mathrm{~Hz}$, contrast 0.36 (range $0.2-0.5$ ) and mean luminance $50 \mathrm{cdm}^{-2}$. The period of GABA application was restricted to the length of time necessary to just cover the duration of the test sequence and we observed immediate recovery of $\mathrm{V} 1$ firing on cessation of drug application. The restricted duration of the application periods and potent GABA uptake mechanisms limited spread. Both our own work and that of others (Herz et al., 1969; Stone, 1985; Grieve and Sillito, 1991, 1995) would predict an effective blockade extending $600 \mu$ from the pipette tip, which would equate to a lateral spread of less than half a degree given the magnification factor pertaining in V1.

We compared responses before, during, and after focal inactivation of the layer 6 cell. Responses were computed from the mean firing rate averaged over the full duration of the stimulus presentation. LGN cells were only considered to show a change in response magnitude if there was a significant difference between response levels evoked during control and V1 drug application conditions $(p<0.05$, paired two-tailed $t$ test) and if the response levels returned to control values following cessation of drug application. To assess LGN and cortical receptive field center to center separation and overlap we used the data from all the stimulus protocols described above to determine the center location and spatial extent of each cell's receptive field center. We fitted the data from the XY patch tests with the best single two-dimensional Gaussian model and the data from the area summation curves with a difference of Gaussian model. Model fitting was performed using the fmincon function (Matlab, MathWorks), minimizing the mean-squared or mean fractional error between the data and the model (Sceniak et al., 2001; Wang et al., 2006). For all cells, we based our judgment of center size on the procedure giving the largest measurement (Jones et al., 2001). Nonparametric two-tailed tests were used for population data comparisons. In 

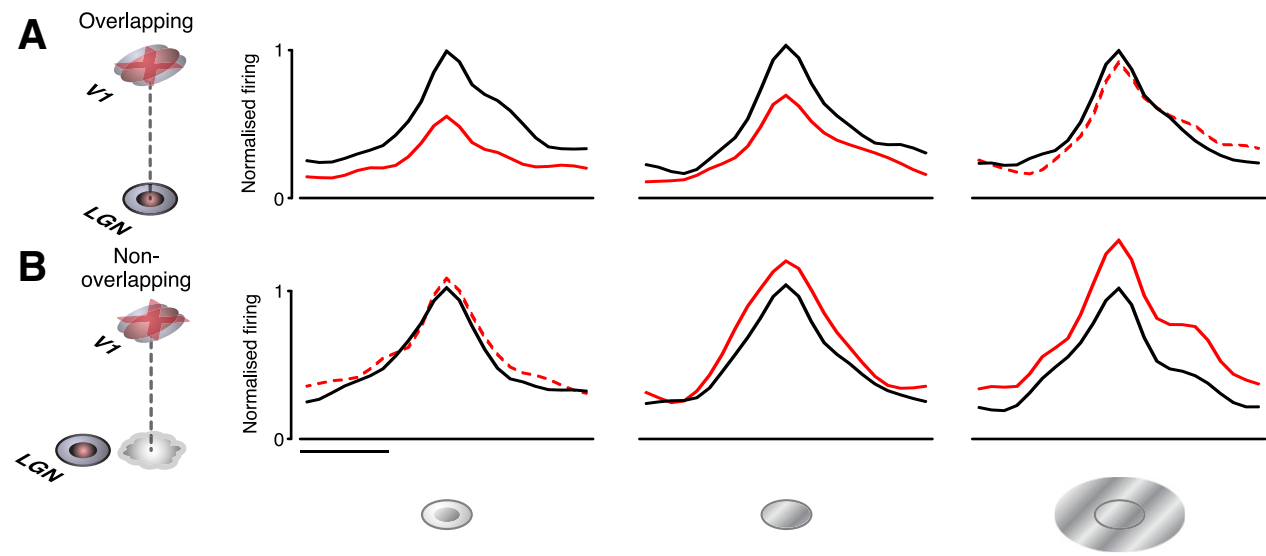

Figure 2. Population peristimulus time histograms (PSTHs) documenting differential effects of focal inactivation of corticothalamic feedback by iontophoretic application of GABA on overlapping $(\boldsymbol{A})$ and nonoverlapping $(\boldsymbol{B})$ LGN cell locations. $\boldsymbol{A}, \boldsymbol{B}$, Wrapped PSTHs of the population averaged responses to smaller than optimal (left), optimal (middle), and larger than optimal (right) diameter stimuli during control (black) and V1 inactivation (red) conditions. Significant response changes (see Results) during V1 inactivation denoted by solid red lines, nonsignificant changes denoted by dotted lines. To compare PSTHs across neurons, individual histograms were shifted by the neuron's latency so that the bin containing the maximal responses was aligned. For each stimulus condition, responses for each neuron were normalized to the control peak response. Histograms were averaged for each group and smoothed. Scale bar, $100 \mathrm{~ms}$.

cases where more than one smaller than optimal diameter stimulus was tested, the smallest diameter tested was always used for summary statistics. For larger than optimal diameter stimuli, we averaged the data from all diameters comprising the plateau phase of the tuning curve.

\section{Results}

We focused on the response to a simple class of visual stimuli, drifting sinusoidal gratings of varying diameter, to check for influences on center and surround mechanisms. Data were obtained from 14 parvocellular cells with receptive fields overlapping the receptive field of the cortical inactivation site and 12 with receptive fields displaced from the inactivation site. The control responses of parvocellular cells to patches of varying diameter centered on their receptive field reveal an increase in response magnitude as diameter increases to a value defined as the "optimal diameter" followed by a decline as the diameter increases further and encroaches on the nonclassical receptive field surround (Wiesel and Hubel, 1966; Webb et al., 2002; Alitto and Usrey, 2008). Having taken control data from the LGN cells we then examined the effect on these responses of reversibly inactivating layer 6 of V1 by iontophoretic application of GABA to remove the corticofugal feedback from that location. We only took data if we both silenced the recorded single cell isolated in layer 6 and the background driving picked up by the electrode.

Focal inactivation of layer 6 affected these responses in a manner that was dependent on whether the receptive fields of the LGN cells overlapped (that is the LGN receptive fields were located within the confines of the cortical receptive field) those of the layer 6 receptive field or not. These data are summarized in Figures 1 and 2 and examples of the data from cells with overlapping receptive fields and nonoverlapping receptive fields are shown in Figures 3 and 4, respectively. The primary observation was simple and clear (Figs. 1, 2). If the cortical and LGN cell receptive fields overlapped, inactivating layer 6 resulted in a decrease in the response of the LGN cell to stimuli at less than the optimal diameter (Figs. $1 B, 3 A-C$ ). The mean reduction was $45.8 \%$ below control levels ( $\pm 5.53 \%$ SEM) and there was a significant difference between control and drug trial responses $(p=$ 0.002 , Wilcoxon matched pairs test). Although the primary effect was on suboptimal diameter stimuli there was also for the whole group of cells, a smaller $(32.3 \pm 8.94 \%$ SEM) but significant $(~ p=$ 0.003 , Wilcoxon) effect at the optimal diameter as well (Figs. $1 B$,
$3 B, C)$. The responses to larger than optimal diameter stimuli were not significantly changed $(2.3 \pm 4.24 \%$ SEM, $p=0.328$, Figs. $1 B, 3 A-C)$.

Where the receptive fields of the layer 6 location were displaced from that of the LGN cells we observed a different pattern of influence. In this case the respective electrode placements used sampled cells that were separated by a mean of 5.7 ( \pm 0.49 SEM) LGN receptive field center sizes (see Materials and Methods). This equated to a mean separation of the centers of the LGN and cortical cell receptive fields of $2.5^{\circ}\left( \pm 0.11^{\circ}\right.$ SEM, range $\left.1.7-3.2^{\circ}\right)$. The primary effect was a significant increase $(46.3 \pm 10.47 \%$ SEM, $p=0.003$ Wilcoxon matched pairs) in response magnitude to supraoptimal sized stimuli and a smaller $(21.6 \pm 7.81 \%$ SEM $)$ but just significant $(p=0.023)$ increase in the response to optimal stimuli (Figs. $1 C, 4 A, B$ ) with no significant influence on the responses to stimuli that were less than the optimal diameter $(p=0.24)$. These effects for both the overlapping and nonoverlapping cells were reversible and repeatable. The postdrug responses returned to control values (Figs. $3 A-C, 4 A, B$ ). For both overlapping and nonoverlapping groups, the variation in magnitude of the effect with stimulus diameter was significant (respectively overlapping, $p=0.0002$ and nonoverlapping, $p=0.005$ Friedman ANOVA).

As described above, it was clear that the pattern of effect evoked by V1 inactivation was highly dependent on the relative locations of the LGN recording and V1 inactivating sites; for overlapping locations, the most notable effect was a decrease in response to smaller than optimal diameter stimuli whereas for nonoverlapping sites, the dominant effect was an increased response to larger than optimal diameter stimuli. Across the data as a whole, there was a highly significant relationship between receptive field overlap and the percentage response reduction/gain during V1 inactivation for smaller than optimal $(r=-0.86, p<$ 0.001 , Spearman Rank order correlation test), optimal ( $r=$ $-0.730, p<0.001)$ and larger than optimal diameter stimuli $(r=-0.80, p<0.001)$. A similar trend was observed for the relationship between center to center separation and the percentage response reduction/gain during V1 inactivation $(r=0.78$, $p<0.001, r=0.55, p<0.005$ and $r=0.63, p<0.001$ for smaller than, optimal and larger than optimal diameters respectively). However, the significant correlations for receptive field separa- 
A

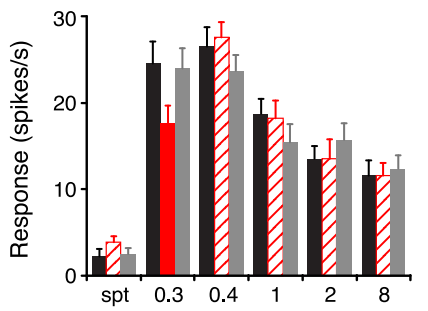

B

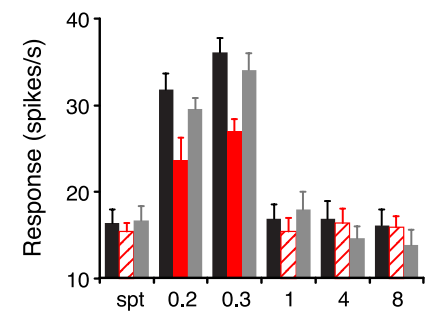

C

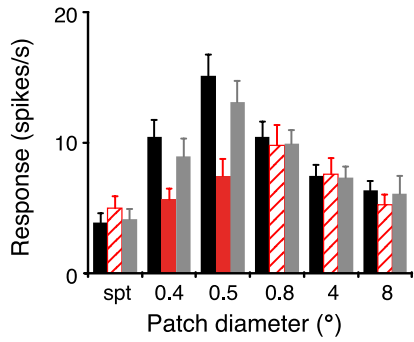

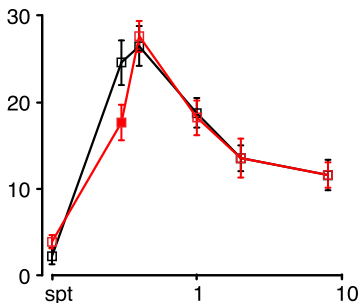
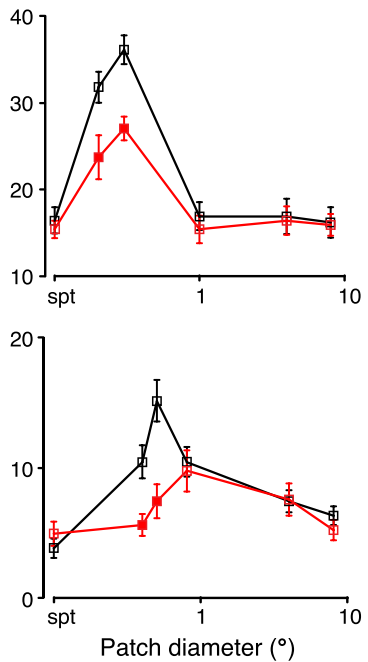
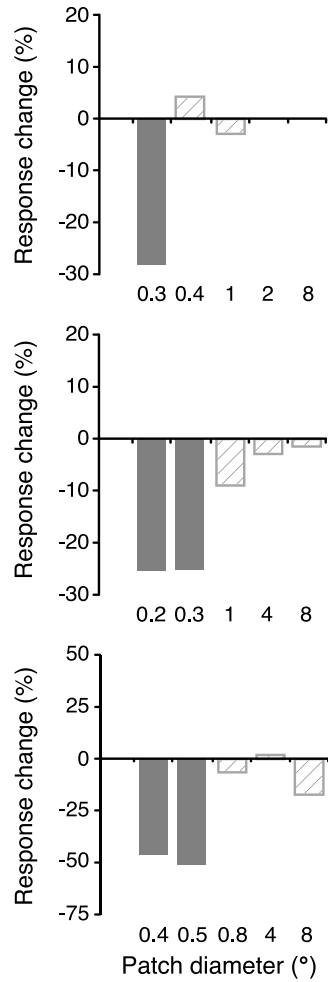

Figure 3. Inactivation of retinotopically overlying $V 1$ feedback reduces responses of $L G N$ cells to smaller than optimal and optimal diameter stimuli. $\boldsymbol{A}-\boldsymbol{C}$, Three representative examples of $L G N$ cell responses for cells whose receptive fields retinotopically overlapped the cortical inactivation site. Records show responses, plotted as both bar histograms (left) and tuning curves (middle), to gratings of varying diameter before (black), during (red), and after (gray) focal GABA inactivation in V1. Responses during V1 inactivation that differed significantly from control levels (see Materials and Methods) are marked by solid red fill (left) and filled symbols (middle). Error bars indicate SEM. Histograms to the right plot the percentage change in response during V1 inactivation, normalized with respect to control values. Significant changes, solid fill; nonsignificant changes, crosshatched. Spt, Spontaneous activity level.

\section{A}

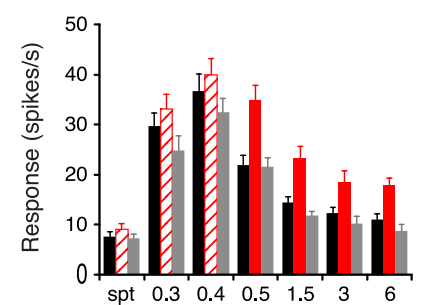

B

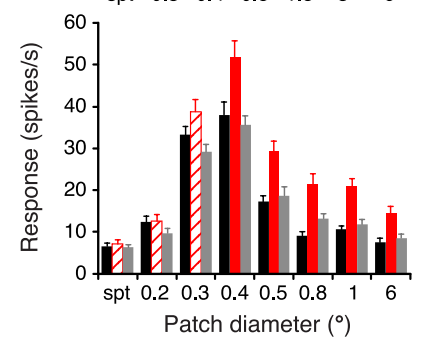

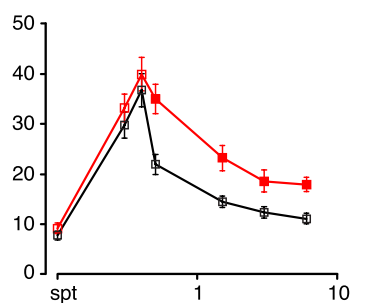

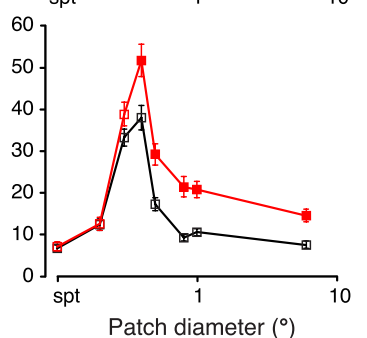

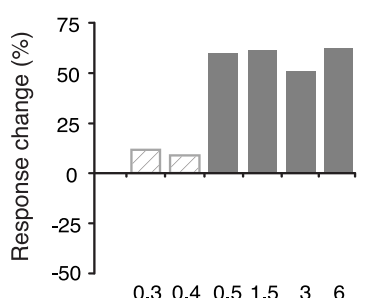

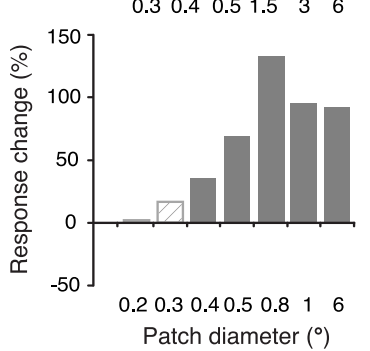

Figure 4. Inactivation of retinotopically displaced V1 feedback enhances responses of $L G N$ cells to optimal and larger than optimal diameter stimuli. $A, B$, Two representative examples of $L G N$ cell responses for cells with receptive fields that did not overlap the cortical inactivation site. Records show responses, plotted as both bar histograms (left) and tuning curves (middle), to gratings of varying diameter before (black), during (red), and after (gray) focal GABA inactivation in V1. Responses during V1 inactivation that differed significantly from control levels (see Materials and Methods) are marked by solid red fill (left) and filled symbols (middle). Error bars indicate SEM. Histograms to the right plot the percentage change in response during V1 inactivation, normalized with respect to control values. Significant changes, solid fill; nonsignificant changes, crosshatched.

tion were essentially linked to the extent of receptive field overlap, and no significant correlations between percentage response change and center to center receptive field separation were observed within the overlapping $(r=-0.06 ; r=-0.35 ; r=-0.28$ respectively for smaller, optimal and larger than optimal, $p>$ 0.05 for each case) and nonoverlapping groups $(r=0.39 ; r=$
$-0.35 ; r=-0.18$ respectively for smaller, optimal and larger than optimal, $p>0.05$ for all) assessed separately.

\section{Discussion}

We assume that our inactivation reveals the presence of a descending influence acting in the opposite direction to the change 
we produced. Thus the data are most compatible with the view that for overlapping receptive fields, the feedback from layer 6 of V1 provides a focused aligned facilitation of LGN cell responses. This enhances the effectiveness of sub optimal and optimal sized stimuli. Conversely, for nonoverlapping stimuli, our observations suggest there is a laterally displaced feedback that increases the surround attenuation of responses to larger than optimal diameter stimuli. The net effect of these two patterns of influence will be to sharpen the spatial focus on stimuli falling within the LGN cell center mechanism and thus the spatial resolution of the receptive field.

It is likely that the facilitatory effects on the responses to small stimuli follow from direct synapses to the relay cells from overlying layer 6 feedback terminals and are mediated by the mGlu1 receptors associated with these terminals (McCormick and von Krosigk, 1992; Godwin et al., 1996; Turner and Salt, 2000). Previous work in the cat has indicated that mGlul receptor activation may enhance the response to receptive field center stimulation (Rivadulla et al., 2002). In the case of the offset enhancement of surround suppression, we suggest that these effects may be mediated via a corticofugal modulation of inhibitory interneurons in the TRN (that pools a larger and displaced, but partially overlapping area of visual space) although an effect on intrinsic inhibitory interneurons cannot be excluded. The fact that both overlying and displaced locations produced effects on optimal diameter stimuli, albeit opposite direction effects suggests that the feedback drives overlapping mechanisms. This might best be conceived by considering a feedback influence on the difference of Gaussian model (Rodieck, 1965) for LGN cell receptive fields. The overlying feedback could provide a nonlinear modulatory enhancement of the excitatory Gaussian underpinning the classical receptive field by direct effects on the relay cells and the displaced feedback could provide a nonlinear modulatory enhancement of the inhibitory Gaussian underpinning the nonclassical surround by effects on intrinsic or TRN inhibitory neurons (Wilson, 1993; Cudeiro and Sillito, 2006; Jones, 2007; Briggs and Usrey, 2011). In this sense then the displaced feedback with inhibitory effect is driven by both stimuli that overly and extend beyond the center mechanism (as in Fig. 4) and thus we presume offsets the effect of the overlying facilitatory mechanism so that this loses effect as the stimuli extend beyond the center mechanism (Fig. 3). On one level our data may seem inimical to the view that parvocellular LGN cell fields are very similar to those of retinal ganglion cells (e.g., Alitto and Usrey, 2008; Briggs and Usrey, 2011) but it is not a logical consequence of our observations. Rather our observations suggest that the LGN cell receptive fields are rephrased in the context of the feedback and local circuit interactions at the level of the thalamus such that the specificity established in the retina is sustained (because the mechanisms offset each other) but modulated and refined by the complexity of the top down mechanisms of cortical origin.

The particular force of our observations here is that they show the presence of a differential feedback influence from the cortex directed to the center and nonclassical surround mechanisms of the LGN cell receptive field. This enhances what might be considered an entirely subcortical facet of the LGN cell responses and reveals that the LGN cell receptive field and center-surround interactions are modulated by the cortical circuit. We are not suggesting that the cortical mechanism in any sense introduces spikes into the LGN cell responses, but specifically that it refines the filter characteristics of the LGN cell receptive field. In so doing it has the implication that when complex stimuli drive the corti- cal circuitry and the feedback from it, the pattern of influence on the LGN cell filters will be correspondingly complex and provide refinements to the filter characteristics that might not be predicted from the simple view of the subcortical mechanism. This is most likely to apply to the feedback from layer 6 in other sensory systems and in this sense reveals the hand of a specific feature of thalamocortical systems. Logically it will also be a mechanism that is further refined when the feedback to the thalamus reflects the cortical focus of attention (O'Connor et al., 2002; McAlonan et al., 2008).

\section{References}

Alitto HJ, Usrey WM (2008) Origin and dynamics of extraclassical suppression in the lateral geniculate nucleus of the macaque monkey. Neuron 57:135-146.

Andolina IM, Jones HE, Wang W, Sillito AM (2007) Corticothalamic feedback enhances stimulus response precision in the visual system. Proc Natl Acad Sci U S A 104:1685-1690.

Briggs F, Usrey WM (2009) Parallel processing in the corticogeniculate pathway of the macaque monkey. Neuron 62:135-146.

Briggs F, Usrey WM (2011) Corticogeniculate feedback and visual processing in the primate. J Physiol 589:33-40.

Cudeiro J, Sillito AM (1996) Spatial frequency tuning of orientationdiscontinuity-sensitive corticofugal feedback to the cat lateral geniculate nucleus. J Physiol 490:481-492.

Cudeiro J, Sillito AM (2006) Looking back: corticothalamic feedback and early visual processing. Trends Neurosci 29:298-306.

de Labra C, Rivadulla C, Grieve K, Mariño J, Espinosa N, Cudeiro J (2007) Changes in visual responses in the feline dLGN: selective thalamic suppression induced by transcranial magnetic stimulation of V1. Cereb Cortex 17:1376-1385.

Godwin DW, Van Horn SC, Eriir A, Sesma M, Romano C, Sherman SM (1996) Ultrastructural localization suggests that retinal and cortical inputs access different metabotropic glutamate receptors in the lateral geniculate nucleus. J Neurosci 16:8181-8192.

Grieve KL, Sillito AM (1991) A re-appraisal of the role of layer VI of the visual cortex in the generation of cortical end inhibition. Exp Brain Res $87: 521-529$

Grieve KL, Sillito AM (1995) Non-length-tuned cells in layers II/III and IV of the visual cortex: the effect of blockade of layer VI on responses to stimuli of different lengths. Exp Brain Res 104:12-20.

Gulyás B, Lagae L, Eysel U, Orban GA (1990) Corticofugal feedback influences the responses of geniculate neurons to moving stimuli. Exp Brain Res 79:441-446.

Herz A, Zieglgänsberger W, Färber G (1969) Microelectrophoretic studies concerning the spread of glutamic acid and GABA in brain tissue. Exp Brain Res 9:221-235.

Jones EG (2007) The thalamus, Ed 2. Cambridge, UK: Cambridge UP

Jones HE, Grieve KL, Wang W, Sillito AM (2001) Surround suppression in primate V1. J Neurophysiol 86:2011-2028.

McAlonan K, Cavanaugh J, Wurtz RH (2008) Guarding the gateway to cortex with attention in visual thalamus. Nature 456:391-394.

McClurkin JW, Marrocco RT (1984) Visual cortical input alters spatial tuning in monkey lateral geniculate nucleus cells. J Physiol 348:135-152.

McCormick DA, von Krosigk M (1992) Corticothalamic activation modulates thalamic firing through glutamate "metabotropic" receptors. Proc Natl Acad Sci U S A 89:2774-2778.

Murphy PC, Sillito AM (1987) Corticofugal feedback influences the generation of length tuning in the visual pathway. Nature 329:727-729.

O'Connor DH, Fukui MM, Pinsk MA, Kastner S (2002) Attention modulates responses in the human lateral geniculate nucleus. Nat Neurosci 5:1203-1209.

Przybyszewski AW, Gaska JP, Foote W, Pollen DA (2000) Striate cortex increases contrast gain of macaque LGN neurons. Vis Neurosci 17:485494.

Rivadulla C, Martínez LM, Varela C, Cudeiro J (2002) Completing the corticofugal loop: a visual role for the corticogeniculate type 1 metabotropic glutamate receptor. J Neurosci 22:2956-2962.

Rodieck RW (1965) Quantitative analysis of cat retinal ganglion cell response to visual stimuli. Vision Res 5:583-601. 
Sceniak MP, Hawken MJ, Shapley R (2001) Visual spatial characterization of macaque V1 neurons. J Neurophysiol 85:1873-1887.

Sillito AM, Cudeiro J, Jones HE (2006) Always returning: feedback and sensory processing in visual cortex and thalamus. Trends Neurosci 29:307-316.

Stone TW (1985) Microiontophoresis and pressure ejection. New York: Wiley.

Tsumoto T, Creutzfeldt OD, Legéndy CR (1978) Functional organization of the corticofugal system from visual cortex to lateral geniculate nucleus in the cat. Exp Brain Res 32:345-364.

Turner JP, Salt TE (2000) Synaptic activation of the group I metabotropic glutamate receptor mGlul on the thalamocortical neurons of the rat dorsal lateral geniculate nucleus in vitro. Neuroscience 100:493-505.

Wang W, Jones HE, Andolina IM, Salt TE, Sillito AM (2006) Functional alignment of feedback effects from visual cortex to thalamus. Nat Neurosci 9:1330-1336.

Webb BS, Tinsley CJ, Barraclough NE, Easton A, Parker A, Derrington AM (2002) Feedback from V1 and inhibition from beyond the classical receptive field modulates the responses of neurons in the primate lateral geniculate nucleus. Vis Neurosci 19:583-592.

Wiesel TN, Hubel DH (1966) Spatial and chromatic interactions in the lateral geniculate body of the rhesus monkey. J Neurophysiol 29:1115-1156.

Wilson JR (1993) Circuitry of the dorsal lateral geniculate nucleus in the cat and monkey. Acta Anat 147:1-13.

Wörgötter F, Nelle E, Li B, Funke K (1998) The influence of corticofugal feedback on the temporal structure of visual responses of cat thalamic relay cells. J Physiol 509:797-815. 